\title{
Similarity and numerical solutions for the Lévêque problem of boundary layer heat and mass transfer in Hagen-Poiseuille flow
}

\author{
Ali Belhocine*, Oday I. Abdullah \\ ${ }^{1}$ Faculty of Mechanical Engineering, University of Sciences and Technology of Oran, LP 1505 El-Mnaouer USTO 31000 , \\ Oran, Algeria \\ ${ }^{2}$ System Technologies and Mechanical Design Methodology, Hamburg University of Technology, Hamburg, Germany
}

Corresponding Author Email: belhocine.2018@gmail.com

https://doi.org/10.18280/ijht.360432

Received: 21 October 2017

Accepted: 28 August 2018

\section{Keywords:}

thermal entrance region, thermal boundary layer, temperature, Nusselt number, Runge-Kutta method

\begin{abstract}
In this paper, the assumptions implicit in Leveque's approximation are re-examined, and the variation of the temperature and the thickness of the boundary layer were illustrated using the developed solution. By defining a similarity variable the governing equations are reduced to a dimensionless equation with an analytic solution in the entrance region. This report gives justification for the similarity variable via scaling analysis, details the process of converting to a similarity form, and presents a similarity solution. The analytical solutions are then checked against numerical solution programming by FORTRAN code obtained via using Runge-Kutta fourth order (RK4) method. Finally, others important thermal results obtained from this analysis, such as; approximate Nusselt number in the thermal entrance region was discussed in detail. After that, a comparison with previously published results on special case of the problem shows excellent agreement
\end{abstract}

\section{INTRODUCTION}

The heat transfer process associated with natural convection is extensively involved in numerous engineering applications due to its diverse applications in geophysics, nuclear reactor system, energy efficient buildings, cooling of electronics system, solar system etc [1]. A heat exchanger is a device used for transferring heat from one fluid to another. The fluid may not be allowed to mix by separating them by a solid wall or they may be in direct contact. They are operated in numerous industries such as power generation, petroleum refineries, chemical and processing plants and HVACs [2]. The experimental studies carried out by the researchers are generally in the field of convective thermal transfers which several authors have addressed in their work, heat transfer problems in a flow of fully developed laminar fluid through circular conduits. An analysis of the heat transfer through a fluid flow and over the boundary layer was established by Hamad and Ferdows [3]. Several researchers have investigated several aspects of the convective flow problems under different flow conditions [4]. However, all the studies above are limited to the Newtonian case in which the classical Navier - Stokes equation is valid [5]. Ambethkar and Kumar [6] used the stream function-vorticity formulation to solve the governing equations of 2-D unsteady viscous incompressible flow along with no-slip and slip wall boundary conditions. This problem has a wide range of applications in engineering and physical sciences. Some of these applications include oil extraction, cooling of electronic devices, heat transfer improvement in heat exchanger devices and drying technologies. Another study was carried out by Wei and Al-Ashhab [7] on boundary layers of a nonNewtonian fluid subject to new boundary conditions. A study was conducted by Trîmbijas et al. [8] to analyze a boundary layer in mixed convection while employing a similarity technique to which partial differential equations are reduced to ordinary differential equations. Ahmed [9] analyzed a boundary layer in natural convection in the presence of transient wall temperatures using the finite difference method. Shen and $\mathrm{Lu} \mathrm{[10]} \mathrm{have} \mathrm{modeled} \mathrm{the} \mathrm{problem} \mathrm{of} \mathrm{free}$ turbulence using the Runge-Kutta method for the prediction of the three-dimensional boundary layer. Mahanthesh et al. [11] carried out a heat flow analysis on the basis of a mathematical model managed by the boundary layer hypotheses while using the similarity method to reduce the governing equations. Eldesoky et al. [12] studied the peristaltic pumping of a compressible fluid in a tube using a perturbation analysis. Baehr and Stephan [13] and Stephan [14] conducted research on heat transfer in the input region with well-specified geometries. Additional work has been done by Asako et al. [15]. Shah and London [16], Kakac et al. [17], Ebadian and Dong [18], and Kakac and Yener [19] on triangular, rectangular and circular geometries. In the literature, we can find other thermal problems performed on other forms of tube geometries such as; the circular channels, the circular and the parallel plate and a rectangular channel. Thanks to these geometries, the thermal problems have been solved easily using analytical methods, of which whose prediction of the thermal transfer of the cylindrical walls was approached on several models. Hausen [20] developed a model to study the Graetz problem inside a circular tube. Churchill and Ozoe [21-22] proposed simple models to develop flux in a circular duct. With the fully developed asymptote, and for the thermal input region. The Leveque solution was combined by Churchill and Ozoe [21-22]. For the Graetz problem, and in order to predict the thermal 
characteristics in an arbitrary form of the tube, models have been developed by Yilmaz and Cihan [23-24]. These two authors developed models for uniform wall flow conditions (H) and a uniform wall temperature $(\mathrm{T})$ in order to predict the fully developed number of Nusselt. These models were fitted to these models with the Leveque generalized solution so that the input offers an approved model along the length of the tube. In the entrance area of the circular duct, two distinct problems must be considered. One assumes the existence of a fully developed hydrodynamic boundary layer while the other problem is more popular with developing thermal boundary layers. In the case of Graetz's classical problem, the velocity distribution is fully developed and the temperature of the fluid tends to propagate fairly rapidly inside the tube. In the input region, the use of the Levèque approach gives us better convergent results in the approximate solution on which we can assume that the velocity gradient is quite linear and the boundary layer is considered thin. Belhocine and Wan Omar [25], Belhocine [26] conducted an analysis to predict the distribution of the dimensionless temperature in a fully developed laminar flow in a cylindrical pipe. Recently, Belhocine and Wan Omar [27] were able to develop the analytical solution of the problem of convective heat transfer within a pipe whose solution obtained is in the forms of the hypergeometric series. Rajput et al. [28] used a similarity solution to analyse, steady two-dimensional laminar MHD forced convection flow of a nanofluid past a moving surface with convective surface boundary condition.

The main objective of this work is to develop an exact solution of the thermal boundary layer at the inlet of a circular pipe for a fully developed laminar flow commonly called the Levèque approximation. The calculation methodology that we have followed is based on the method of solution in similarity of the variables in order to predict the dimensionless temperature as well as the thickness of the thermal boundary layer near the entrance of the flow. Several steps have been discussed here on the governing equation of the temperature field to reach the solution such that; the nondimensionalization and the use similarity variables, the transform the PDE to a set of PDE's. Summarization of the boundary conditions and the integration of the equation. We then compare the exact approximate solution of the levèque problem, with the numerical results using a Runge-Kutta fourth order (RK4) algorithm implemented by the FORTRAN code. The profiles of the solutions are provided from which we infer that the numerical and exact solutions agreed very well. Another result that we obtained from this study is the number of Nusselt in the thermal entrance region to which a parametric study was carried out and discussed well for the impact of the scientific contribution. Finally, previous results in the literature relating to the values of the Nusselt number in the thermal entrance region have been used in the present work to validate the accuracy of the results of the analytical model.

\section{THE HEAT DIFFUSION EQUATION GOVERNING}

The total thermal energy balance, which is based on the use of equations of continuity and momentum, is simplified by the expression obtained by Bird, Stewart, and Lightfoot [29] is as follows;

$$
\begin{aligned}
& \rho C_{p}\left(\frac{\partial T}{\partial t}+u_{r} \frac{\partial T}{\partial r}+\frac{u_{\theta}}{r} \frac{\partial T}{\partial \theta}+u_{z} \frac{\partial T}{\partial z}\right)= \\
& k\left[\frac{1}{r} \frac{\partial}{\partial r}\left(r \frac{\partial T}{\partial r}\right)+\frac{1}{r^{2}} \frac{\partial^{2} T}{\partial \theta^{2}}+\frac{\partial^{2} T}{\partial z^{2}}\right]+ \\
& 2 \mu\left\{\left(\frac{\partial u_{r}}{\partial r}\right)^{2}+\left[\frac{1}{r}\left(\frac{\partial u_{\theta}}{\partial \theta}+u_{r}\right)\right]^{2}+\left(\frac{\partial u_{z}}{\partial z}\right)^{2}\right\}+ \\
& 2 \mu\left\{\left(\frac{\partial u_{\theta}}{\partial z}+\frac{1}{r} \frac{\partial u_{z}}{\partial \theta}\right)^{2}+\left(\frac{\partial u_{z}}{\partial r}+\frac{\partial u_{r}}{\partial z}\right)^{2}\right\} \\
& +\mu\left[\frac{1}{r} \frac{\partial u_{r}}{\partial \theta}+r \frac{\partial}{\partial r}\left(\frac{u_{\theta}}{r}\right)\right]^{2}
\end{aligned}
$$

\section{THE GRAETZ-POISEUILLE FLOW PROBLEM}

The Graetz problem consists of determining the temperature in a steady state of a fluid passing through a circular pipe whose flow is laminar fully developed. Thus, it is a transfer of heat by convection of a fluid approaching the inlet section of a cylindrical tube with a constant temperature $T_{0}$ whose wall is subjected to a constant temperature $T_{\omega}$. The geometry of the problem is shown in Fig.1.

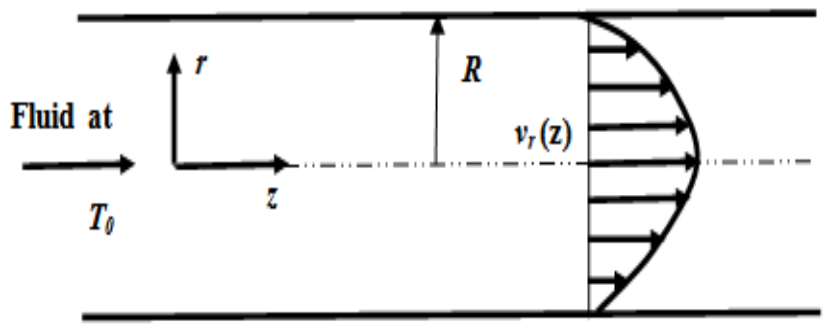

$$
T(R, z)=T_{\omega}
$$

Figure 1. Illustration of Graetz problem

The contour of the velocity of the flow becomes a stable contour after a certain distance from the hydrodynamic inlet and it remains practically fully parabolic and invariable along the circular tube. Our context for solving the thermal problem is to find the behavior of the temperature field as it evolves to be uniform at the temperature of the downstream wall. The distribution of the velocity of the flow is not subordinated by the variation of the temperature as long as the nature of the fluid is incompressible.

- The fluid flow is completely laminar in steady state and fully developed

- The flow is considered incompressible Newtonian whose properties $\rho, \mu, C_{p}, k$. are constant and do not depend on temperature.

- The temperature does not depend on the angular coordinate $\theta\left(\frac{\partial T}{\partial \theta}=0\right)$,

- Negligible viscous dissipation

The expression of the velocity of a fully developed flow is given by the following form [27]: 


$$
u_{z}=v_{0}\left[1-\left(\frac{r}{R}\right)^{2}\right]
$$

where $v_{0}$ is the maximum speed (center of the tube), $u_{r}=0$, and $u_{\theta}=.0$. The energy equation is subject to the assumptions mentioned above, Eq.(1) can be written as follows [30]:

$$
v_{0}\left(1-\frac{r^{2}}{R^{2}}\right) \frac{\partial T}{\partial z}=\alpha\left[\frac{1}{r} \frac{\partial}{\partial r}\left(r \frac{\partial T}{\partial r}\right)+\frac{\partial^{2} T}{\partial z^{2}}\right]
$$

where $\alpha=k / \rho C_{p}$ is called the thermal diffusivity which has dimensions $\left(\mathrm{m}^{2} / \mathrm{s}\right)$, our problem is subjected to the following boundary conditions; at the inlet of the tube $\mathrm{T}(\mathrm{r}, 0)=\mathrm{T}_{0}$; at the wall of the tube $T(R, z)=T_{\omega}$ and at the centerline $T(0, z)$ is finite or $\frac{\partial T}{\partial r}(0, z)=0$.

Consider the following dimensionless terms [30]:

$\left\{\begin{array}{l}\theta=\frac{T-T_{\omega}}{T_{0}-T_{\omega}} \\ Y=\frac{r}{R} \\ Z=\frac{z}{R P e}\end{array}\right.$

where the Péclet Number $\mathrm{Pe}=\frac{\mathrm{Rv}_{0}}{\alpha}$

By substituting the variables $T, r, z$ for their expressions as a function of the dimensionless variables $\theta, Y, Z$ in the heat equations, we obtain the following equations [30]:

$\left(1-Y^{2}\right) \frac{\partial \theta}{\partial Z}=\frac{1}{Y} \frac{\partial}{\partial Y}\left(Y \frac{\partial \theta}{\partial Y}\right)+\frac{1}{P e^{2}} \frac{\partial^{2} \theta}{\partial Z^{2}}$

$\theta(Y, 0)=1, \theta(1, Z)=0, \frac{\partial \theta}{\partial Y}(0, Z)=0$

The influence of the axial diffusion is totally neglected when we apply the assumptions of the boundary layer, which implies the resolution of the following dimensionless equation [30]

$$
\left(1-Y^{2}\right) \frac{\partial \theta}{\partial Z}=\frac{1}{Y} \frac{\partial}{\partial Y}\left(Y \frac{\partial \theta}{\partial Y}\right)
$$

This equation can be solved by the technique of the separations of the variables at which the temperature that we seek will be found in terms of hypergeometic series [30]

$$
\theta(Y, Z)=\sum_{n=1}^{\infty} C_{n} e^{-\beta_{n}^{2}} G_{n}(Y)
$$

where $\beta_{n}$, and $G_{n}(Y)$, are respectively the eigenvalues and the eigenfunctions associated with the Sturm-Liouville problems. The coefficients $C_{n}$ can be obtained by using the orthogonality property of the eigenfunctions defined as follows [30]:

$$
C_{n}=\frac{\int_{0}^{1} G_{n}(Y) Y\left(1-Y^{2}\right) d Y}{\int_{0}^{1} G_{n}^{2}(Y) Y\left(1-Y^{2}\right) d Y}
$$

\section{THE LÉVÊQUE APPROXIMATION}

For all values of the axial position, the orthogonal function expansion solution obtained in the resolution of the classical Graetz problem is quite convergent, but the convergence is very slow as soon as one approaches the input tube. Indeed, for very large values of $Z$, the factor $e^{-\lambda_{n}^{2} Z}$ has become converged. Lévêque [31] examined the thermal entrance zone in a cylindrical pipe while developing an approximate solution which is formally advantageous when the orthogonal function tends towards convergence too gradually (Fig.2).

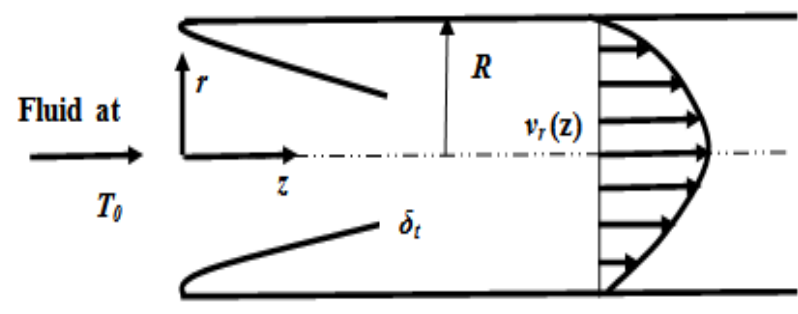

$T(R, z)=T_{\omega}$

Figure 2. Simplifying representation of the Lévêque approximation

According to Lévêque's assumption, we can take the thickness of the boundary layer $\delta_{t}\langle\langle R$, which leads to the following simplifications:

- In the radial conduction term, we can neglect here the effects of curvature. Thus, derivative $\frac{1}{\mathrm{r}} \frac{\partial}{\partial \mathrm{r}}\left(\mathrm{r} \frac{\partial \mathrm{T}}{\partial \mathrm{r}}\right)$ is approximated by $\frac{1}{\mathrm{R}} \frac{\partial}{\partial \mathrm{r}}\left(\mathrm{R} \frac{\partial \mathrm{T}}{\partial \mathrm{r}}\right)=\frac{\partial^{2} \mathrm{~T}}{\partial \mathrm{r}^{2}}$

- We are interested in the thermal boundary layer of the velocity allocation of which it can be developed in a Taylor series from the wall of the pipe according to a measured position, if we keep the first non-zero term

If we set $x=R-r$, the speed distribution will take the following form:

$v_{z}(r)=v_{0}\left(1-\frac{(R-r)^{2}}{R^{2}}\right)=v_{0}\left(2 \frac{x}{R}-\frac{x^{2}}{R^{2}}\right) \approx 2 v_{0} \frac{x}{R}$

- Let us know, the boundary conditions of the flow entering the pipe are those that lie outside the boundary layer, we will exploit the boundary condition $T(x \rightarrow \infty) \rightarrow T_{0}$ instead of that market at the center of the tube to arrive at the Graetz solution.

\subsection{Governing Lévêque's equation}

Starting from the reduced energy equation whose axial conduction has been neglected yet, and considering the said 
hypotheses, for the temperature field, we obtain the following governing equation

$2 v_{0} \frac{x}{R} \frac{\partial T}{\partial z}=\alpha \frac{\partial^{2} T}{\partial x^{2}}$

Using the string rule, in order to convert the second derivative of $r$ into that of $x$, we get

$X \frac{\partial \theta}{\partial Z}=\frac{\partial^{2} \theta}{\partial X^{2}}$

\subsection{Boundary conditions}

The temperature $T(x, z)$,is controlled by boundary conditions which are fixed like this.

$\left\{\begin{array}{l}T(x, 0)=T_{0} \\ T(0, z)=T_{\omega} \\ T(\infty, z)=T_{0}\end{array}\right.$

\subsection{Non-dimensionalization}

Now, we will use dimensionless variables for the simplification of the equation. For this, we introduce the temperature and the axial coordinate of the following forms

$\theta=\frac{T-T_{\omega}}{T_{0}-T_{\omega}}, Z=\frac{z}{R P e}$

The scaled governing equation placed on the wall by $X=x / R$ and boundary conditions are given as follows

$2 X \frac{\partial \theta}{\partial Z}=\frac{\partial^{2} \theta}{\partial X^{2}}$

$\left\{\begin{array}{l}\theta(X, 0)=1 \\ \theta(0, Z)=0 \\ \theta(\infty, Z)=1\end{array}\right.$

\section{ANALYTICAL METHODOLOGY FOR PROBLEM SOLVING: TEMPERATURE FIELD AND THERMAL BOUNDARY LAYER}

At the current problem, we are looking for a similarity solution for the temperature field, we assume, $\theta(X, Z)=F(\eta)$, where $\eta=\mathrm{X} / \delta(\mathrm{Z})$ is the similarity variable and $\delta(\mathrm{Z})$ is an ignored variable that provides us with the thickness of the thermal boundary layer. Using the chain rule, we will perform the following necessary transformations.

$\frac{\partial \theta}{\partial Z}=\frac{\partial \eta}{\partial Z} \frac{d F}{d \eta}=\left(-\frac{X d \delta}{\delta^{2} d Z}\right) \frac{d F}{d \eta}=-\frac{\eta d \delta}{\delta d Z} \frac{d F}{d \eta}$

$\frac{\partial \theta}{\partial X}=\frac{\partial \eta}{\partial X} \frac{d F}{d \eta}=\frac{1}{\delta} \frac{d F}{d \eta}$ $\frac{\partial^{2} \theta}{\partial X^{2}}=\frac{\partial}{\partial X}\left[\frac{1}{\delta(Z)} \frac{d F}{d \eta}\right]=\frac{1}{\delta} \frac{\partial}{\partial X}\left[\frac{d F}{d \eta}\right]=$

$\frac{1}{\delta} \frac{\partial \eta}{\partial X} \frac{d}{d \eta}\left[\frac{d F}{d \eta}\right]=\frac{1}{\delta^{2}} \frac{d^{2} F}{d \eta^{2}}$

Using the important results, the previous equation for $\theta(X$, $Z$ ), has been reduced to the solution of the ordinary differential equation for $F(\eta)$

$\frac{d^{2} F}{d \eta^{2}}+2 \eta^{2}\left(\delta^{2} \frac{d \delta}{d Z}\right) \frac{d F}{d \eta}=0$

We put the term in parentheses $\left(\delta^{2} \frac{\mathrm{d} \delta}{\mathrm{dz}}\right)$ a constant is equal to $3 / 2$ because it is authentic that the supposition of similarity will lose unless this magnitude is indispensable to be independent of Z. Finally, we get at the solution of a system of equations composed of two unknowns to be found $F(\eta)$ and $\delta(\mathrm{Z})$.

$$
\left\{\begin{array}{l}
\frac{d^{2} F}{d \eta^{2}}+3 \eta^{2} \frac{d F}{d \eta}=0 \\
\delta^{2} \frac{d \delta}{d Z}=\frac{3}{2}
\end{array}\right.
$$

Starting from the boundary condition on $\theta(X, Z)$, we can calculate the derivatives the boundary conditions of these formulas. We notice that $\theta(0, Z)=0$ which implies $\theta(\infty, Z)=1$ and $\mathrm{F}(0)=0$ which tends to $F(\infty)=1$. The residual clause at the entrance of the tube gives us:

$\theta(X, 0)=F\left(\frac{X}{\delta(0)}\right)$

By favoring $\delta(0)=0$, this condition has been dismantled in the one obtained recently $F(\infty)=1$, when the variable $X$ tends towards the infinite, that is to say $X \rightarrow \infty$. By joining the two boundary conditions on $\mathrm{F}(\eta)$ and $\delta(\mathrm{Z})$, we get to:

$F(0)=0, F(\infty)=1$ and $\delta(0)=0$

From the equation, we can write

$\frac{\frac{d^{2} F}{d \eta^{2}}}{\frac{d F}{d \eta}}=-3 \eta^{2}$

By integrating the two terms of the obtained equation

$\frac{\frac{d^{2} F}{d \eta^{2}}}{\frac{d F}{d \eta}}=-\int 3 \eta^{2} d \eta$

We arrive at the following expression 
$\ln \left[\frac{d F}{d \eta}\right]=-\eta^{3}+k=\ln \left[C e^{-\eta^{3}}\right]$

where $\mathrm{k}$ and $\mathrm{C}$ are constants of the integral

By analogy, the following is drawn

$\frac{d F}{d \eta}=C e^{-\eta^{3}}$

Finally, the solution of the equation will take the following expression:

$F(\eta)=C \int_{0}^{\eta} e^{-\eta^{3}} d \eta$

The function $F(\eta)$ checks the initial condition for $\eta=$ $0, F(0)=0$, and also considers the boundary condition for $\eta \rightarrow \infty, \mathrm{F}(\infty)=1$; which implies

$F(\infty)=1=C \int_{0}^{\infty} e^{-\eta^{3}} d \eta$

From where

$$
C=\frac{1}{\int_{0}^{\infty} e^{-\eta^{3}} d \eta}
$$

So

$F(\eta)=\frac{\int_{0}^{\eta} e^{-\eta^{3}} d \eta}{\int_{0}^{\infty} e^{-\eta^{3}} d \eta}$

The scaled boundary layer thickness $\delta(\mathrm{Z})$ is calculated by the integration, which gives the following solution

$\delta(Z)=\left(\frac{9}{2} Z\right)^{1 / 3}$

Finally, the solution of our differential equation takes the following form

$F(\eta)=\frac{\int_{0}^{\eta} e^{-\gamma^{3}} d \gamma}{\int_{0}^{\infty} e^{-\gamma^{3}} d \gamma}=\frac{1}{\Gamma(4 / 3)} \int_{0}^{\eta} e^{-\gamma^{3}} d \gamma$

where $\Gamma(x)$ is the Gamma function [32], a MATLAB code was used to approximate the values of the integral and the function $F(\eta)$ for each abscissa $\eta$.

\section{NUMERICAL RESOLUTION OF THE PROBLEM USING RK04 METHOD}

The original ODE of our problem is defined as follows:

$$
\frac{d^{2} F}{d \eta^{2}}+3 \eta^{2} \frac{d F}{d \eta}=0
$$

With $\eta=0, F(0)=0$ and $\frac{d F}{d \eta}(0) \neq 0$

We will use the fourth-order Runge-Kutta method, so we have the following system:

$\left\{\begin{array}{l}\frac{d F}{d \eta}=P \\ \frac{d P}{d \eta}=-3 \eta^{2} P\end{array}\right.$

With $\mathrm{F}(0)=0$ and $\mathrm{P}(0)=1$

If we have two ordinary differential equations of the first order, we have:

$\left\{\begin{array}{l}\frac{d y}{d x}=f(x, y, z) \\ \frac{d z}{d x}=g(x, y, z)\end{array}\right.$

By applying the RK04 method on this system, we give:

$\left\{\begin{array}{l}y_{i+1}=y_{i}+\left(k_{0}+2 k_{1}+2 k_{2}+k_{3}\right) / 6 \\ z_{i+1}=z_{i}+\left(l_{0}+2 l_{1}+2 l_{2}+l_{3}\right) / 6\end{array}\right.$

where

$\left\{\begin{array}{l}k_{0}=h f\left(x_{i}, y_{i}, z_{i}\right) \\ k_{1}=h f\left(x_{i}+\frac{h}{2}, y_{i}+\frac{k_{0}}{2}, z_{i}+\frac{l_{0}}{2}\right) \\ k_{2}=h f\left(x_{i}+\frac{h}{2}, y_{i}+\frac{k_{1}}{2}, z_{i}+\frac{l_{1}}{2}\right) \\ k_{3}=h f\left(x_{i}+h, y_{i}+k_{2}, z_{i}+l_{2}\right)\end{array}\right.$

and

$\left\{\begin{array}{l}l_{0}=h g\left(x_{i}, y_{i}, z_{i}\right) \\ l_{1}=h g\left(x_{i}+\frac{h}{2}, y_{i}+\frac{k_{0}}{2}, z_{i}+\frac{l_{0}}{2}\right) \\ l_{2}=h g\left(x_{i}+\frac{h}{2}, y_{i}+\frac{k_{1}}{2}, z_{i}+\frac{l_{1}}{2}\right) \\ l_{3}=h g\left(x_{i}+h, y_{i}+k_{2}, z_{i}+l_{2}\right)\end{array}\right.$

We have adopted the Kutta Runge algorithm for finding the solution of our system of equations;

- The interval for the integration of the equations is chosen to perform our calculations: $[\mathrm{a}, \mathrm{b}]$ if we take $\mathrm{a}=0$, and $\mathrm{b}=3$

- The number of iterations $\mathrm{N}=30$,

- The size of the iterations will be estimated as follows: $\mathrm{h}=(\mathrm{b}-\mathrm{a}) / \mathrm{N}=3 / 30=0.1$

The flowchart for the above process is shown in Figure 3. 


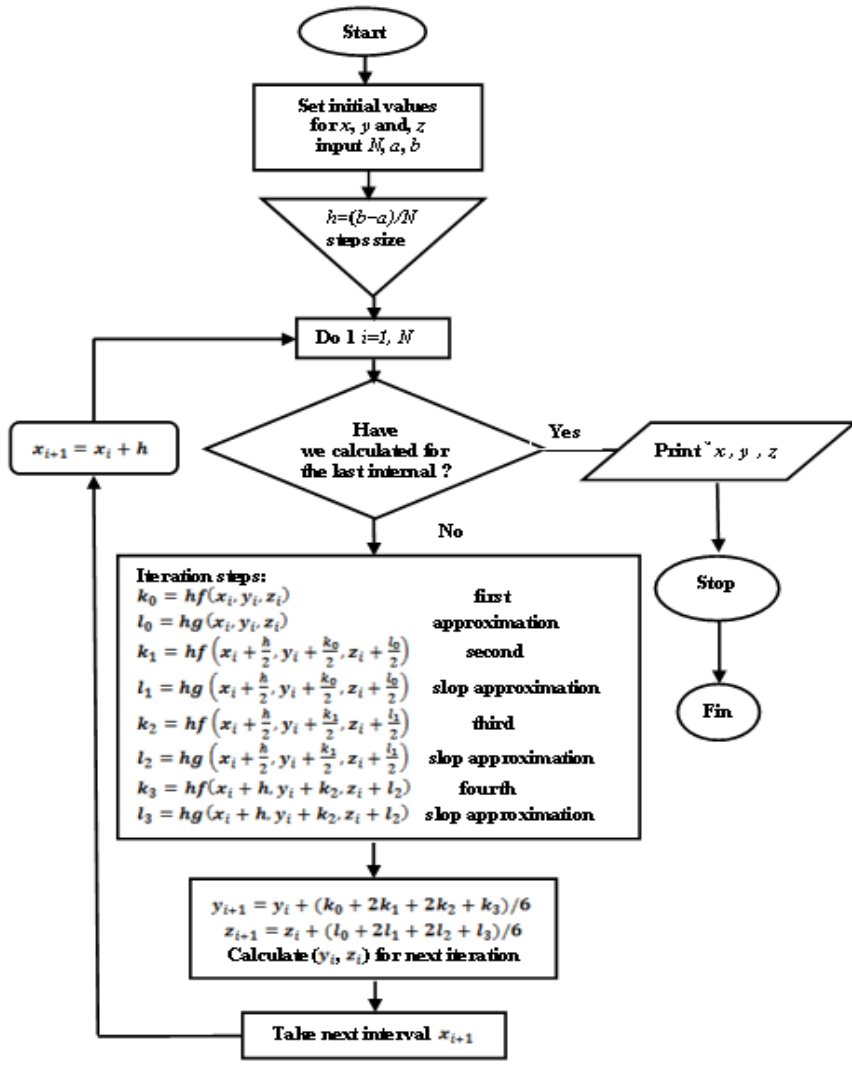

Figure 3. Flowchart of the RK-4 method for resolving the second ODE's systems

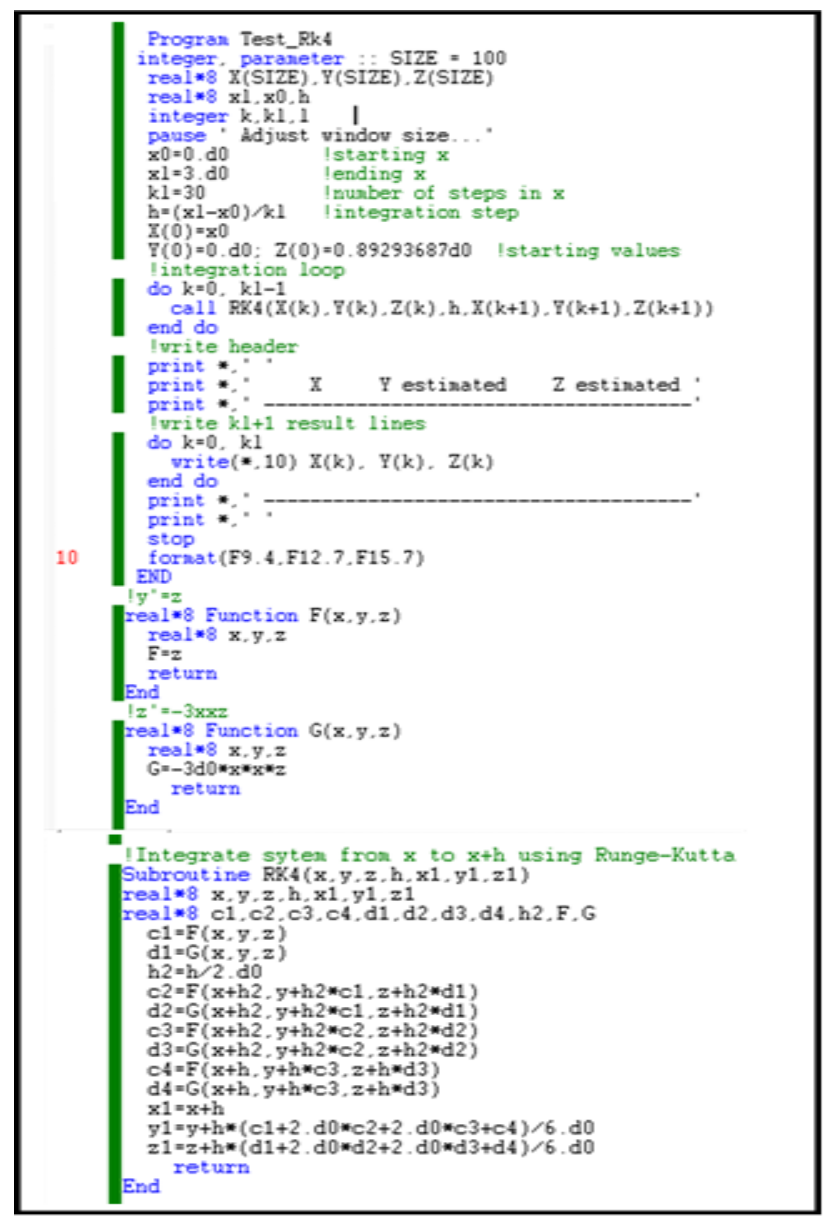

Figure 4. FORTRAN code of Runge Kutta for set of first order differential equations
The main program was drafted by FORTRAN, which will solve the problem of Levèque whose procedure initiated to solve simultaneously two differential equations of the order by the method of Runge Kutta RK04. This program relies on a definition of two functions whose subroutine RK04 is called at each repetition of the loop that intervenes in the calculations. The code edited in the machine that was executed is illustrated in detail in Figure 4.

\section{RESULTANTS AND DISCUSSIONS}

\subsection{Validation of the numerical results via the analytical solution of the problem}

The analytical solution that we have developed above is compared here with the numerical results derived from the FORTRAN V.05 calculation code. The results of the two methods are condensed in detail in Table 1.

Table 1. Exact results and the numerical solution

\begin{tabular}{|c|c|c|c|}
\hline \multicolumn{2}{|c|}{$\begin{array}{c}\text { Exact analytical } \\
\text { solution }\end{array}$} & \multicolumn{2}{|c|}{$\begin{array}{l}\text { Numerical solution via fourth-order } \\
\text { Runge-Kutta (RK4) method using a } \\
\text { FORTRAN Code }\end{array}$} \\
\hline$\eta$ & $F(\eta)=\theta(X, Z)$ & $\mathrm{X} \quad \mathrm{Y}$ estimated $\mathrm{Z}$ & estimated \\
\hline 0 & 0 & $0.0000 \quad 0.0000000$ & 0.8929367 \\
\hline 0,1 & 0,08927136 & $0.1000 \quad 0.0892714$ & 0.8920442 \\
\hline 0,2 & 0,17823109 & $0.2000 \quad 0.1782309$ & 0.8858217 \\
\hline 0,3 & 0,26608715 & $0.3000 \quad 0.2660866$ & 0.8691499 \\
\hline 0,4 & 0,35156264 & $0.4000 \quad 0.3515626$ & 0.8375790 \\
\hline 0,5 & 0,43300027 & $\begin{array}{lll}0.5000 & 0.4329998\end{array}$ & 0.7880137 \\
\hline 0,6 & 0,50853023 & $0.6000 \quad 0.5085291$ & 0.7194705 \\
\hline 0,7 & 0,57631574 & $0.7000 \quad 0.5763146$ & 0.6336619 \\
\hline 0,8 & 0,63483615 & $0.8000 \quad 0.6348343$ & 0.5351335 \\
\hline 0,9 & 0,68314582 & $0.9000 \quad 0.6831438$ & 0.4307465 \\
\hline 1 & 0,72105634 & $1.0000 \quad 0.7210538$ & 0.3284985 \\
\hline 1,1 & 0,74916957 & $1.1000 \quad 0.7491656$ & 0.2359384 \\
\hline 1,2 & 0,76875346 & $1.2000 \quad 0.7687482$ & 0.1586460 \\
\hline 1,3 & 0,78149478 & $1.3000 \quad 0.7814872$ & 0.0992794 \\
\hline 1,4 & 0,78918921 & $\begin{array}{ll}1.4000 & 0.7891808\end{array}$ & 0.0574867 \\
\hline 1,5 & 0,79347888 & $\begin{array}{ll}1.5000 & 0.7934697\end{array}$ & 0.0306282 \\
\hline 1,6 & 0,79567283 & $1.6000 \quad 0.7956641$ & 0.0149366 \\
\hline 1,7 & 0,79669613 & $1.7000 \quad 0.7966892$ & 0.0066372 \\
\hline 1,8 & 0,79712921 & $\begin{array}{ll}1.8000 & 0.7971242\end{array}$ & 0.0026785 \\
\hline 1,9 & 0,7972944 & $1.9000 \quad 0.7972914$ & 0.0009805 \\
\hline 2 & 0,79735155 & $2.0000 \quad 0.7973494$ & 0.0003264 \\
\hline 2,1 & 0,79736852 & $2.1000 \quad 0.7973676$ & 0.0000995 \\
\hline 2,2 & 0,79737298 & $2.2000 \quad 0.7973729$ & 0.0000283 \\
\hline 2,3 & 0,79737387 & $2.3000 \quad 0.7973742$ & 0.0000077 \\
\hline 2,4 & 0,79737387 & $2.4000 \quad 0.7973746$ & 0.0000021 \\
\hline 2,5 & 0,79737477 & $2.5000 \quad 0.7973747$ & 0.0000006 \\
\hline 2,6 & 0,79737477 & $2.6000 \quad 0.7973747$ & 0.0000002 \\
\hline 2,7 & 0,79737477 & $2.7000 \quad 0.7973747$ & 0.0000001 \\
\hline 2,8 & 0,79737477 & $2.8000 \quad 0.7973747$ & 0.0000000 \\
\hline 2,9 & 0,79737477 & $2.9000 \quad 0.7973747$ & 0.0000000 \\
\hline 3 & 0,79737477 & $3.0000 \quad 0.7973747$ & 0.0000000 \\
\hline
\end{tabular}

Fig. 5 shows a comparison between the resolution results of the equation predicted by the analytical method and the numerical data derived from the FORTRAN code, the two sets of results of which are plotted in the same figure. On the basis of Fig. 5, it can be seen that the two curves are fairly identical, while observing that the dimensionless temperature $\theta$ gradually and gradually increases to the abscissa $Z=0.7$, 
then loops and arches a little, by varying its path until it reaches the position $Z=1.7$ where it stabilizes at a constant value 0.79 along the tube until the outlet of the fluid stream. In the same figure, the derivative function $\frac{\mathrm{dF}}{\mathrm{d} \eta}$ which is physically interpreted as the variation of the thermal transfer coefficient (h) indicated in blue color which is a solution of our set equations decreases exceptionally as it moves away from the inlet region and then reaches the value zero on the abscissa $Z=1.6$ until the exit of the flow. Fig. 5 shows clearly that the results of the analysis solution are very excellent convergence with those of the numerical results performed by the Visual FORTRAN v5.0 calculation code during which the use of the RK04 method obviously gives us a severely accurate assessment.

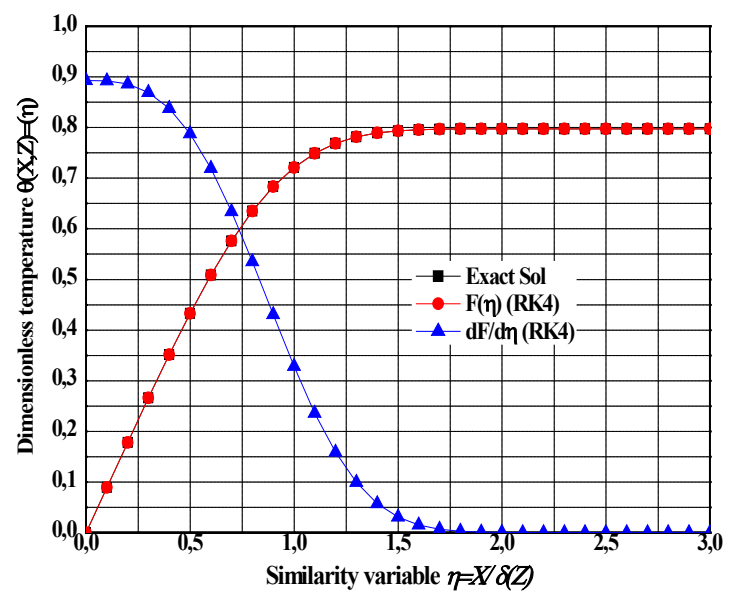

Figure 5. Comparison of exact and fourth-order Runge Kutta (RK4) numerical solutions

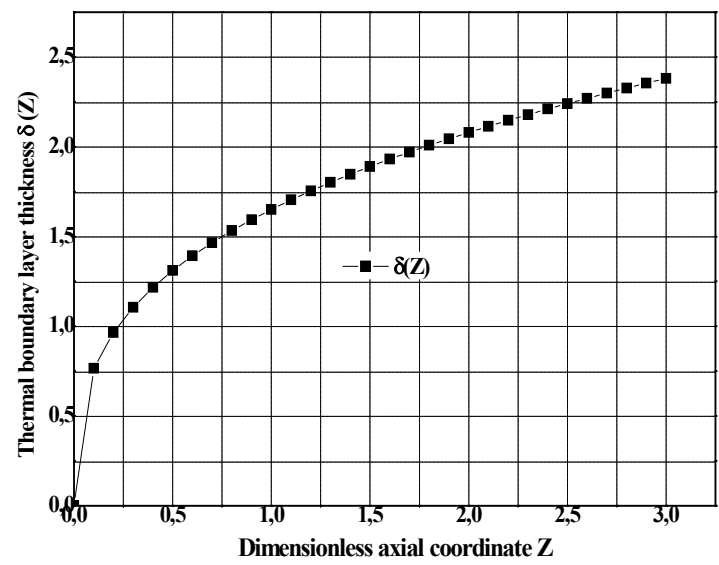

Figure 6. Thermal boundary layer thickness distribution by analytical method

Fig. 6 shows the variation in the thickness of the thermal boundary layer as a function of the longitudinal coordinate where the latter increases slowly from the zero position towards the direction of flow of the fluid as it penetrates the pipe through its center and arrogates its total space. At the inlet of the tube and its wall, the shear stress is greater during which the thickness of the boundary layer is very short and slowly decreases to the fully developed value. In fact, the collapse of the pressure is increased in the inlet zone of the tube under the effect which may cause the phenomenon of friction over the whole of the tube. This elevation can be negligible for long and important tubes in short lengths. A thin layer can be observed on the wall at which the velocity of flow is less than the wall. By going from front to back, the thickness of the thermal boundary layer lengthens along the channel.

\subsection{The heat transfer coefficient}

Depending on the axial position, we try to understand the heat fluxes of the wall when a fluid flow is involved, we can calculate it directly using the following formula:

$q_{\omega}(z)=k \frac{\partial T}{\partial r}(R, z)$

By usual notation, the convective transfer coefficient $h(z)$ is known from the following expression

$q_{\omega}(z)=h(z)\left(T_{\omega}-T_{b}\right)$

where $T_{b}$ is the bulk or cup-mixing average temperature.

The average bulk temperature is mathematically defined as:

$T_{b}=\frac{\int_{0}^{R} 2 \pi r V(r) T(r, z) d r}{\int_{0}^{R} 2 \pi r V(r) d r}$

where $V(r)=v_{0}\left(1-r^{2} / R^{2}\right)$ is the velocity field. The temperature gradient at the wall is commonly subordinate to the heat exchange coefficient; we can estimate it as follows:

$h(z)=\frac{k \frac{\partial T}{\partial r}(R, z)}{\left(T_{\omega}-T_{b}\right)}$

The Nusselt number is defined as a dimensionless heat exchange coefficient.

$N u(Z)=\frac{2 h R}{k}=-2 \frac{\frac{\partial \theta}{\partial Y}(1, Z)}{\theta_{b}(Z)}$

where $\theta_{\mathrm{b}}$ is the without dimensional bulk temperature along pipe.

We approximate the mean temperature $T_{b}$ by the temperature of the liquid entering the pipe $T_{0}$ and this, in the region of entry where the boundary layer is thin.

As a result, and through the thermal input region, the heat exchange coefficient $(\mathrm{h})$ is expressed as

$q_{\omega}=k \frac{\partial T}{\partial r}(R, z)=h\left(T_{\omega}-T_{0}\right)$

We know the Nusselt number $N u=2 h R / k$, and by introducing the dimensionless variables, we obtain the following: 
$N u(Z)=2 \frac{\partial \theta}{\partial X}(0, Z)=\frac{2}{\delta(Z)} \frac{\partial F}{\partial \eta}(0)$

By substituting $\delta(Z)$ and $\frac{\partial F}{\partial \eta}(0)$, the final formula of the Nusselt number as a function of the variables Pe, Z, and R which we evaluated in the input region can be expressed as follows:

$$
N u(Z) \approx 1.357 P e^{1 / 3}\left(\frac{R}{z}\right)^{1 / 3}
$$

By comparing with the exact solution, we can now appreciate that this calculation is a better evaluation in the range

$$
\frac{P e}{2500} \leq\left(\frac{z}{R}\right) \leq \frac{P e}{50}
$$

Fig. 7 shows the variation obtained in the input region of the Nusselt number as a function of the axial distance $Z$ obtained in the thermal input region for various radius of the pipe. We can observe that the number of Nusselt, $\mathrm{Nu}(\mathrm{Z})$, rises as a function of the increase of the radius of the tube and that this influence is very noticeable enlarged at the entrance. When $\mathrm{Z}$ is greater than a certain distance, all the bundles of curves have become intensified and they stabilize horizontally flat, this explains why the fully developed boundary layer is reached. Indeed, the boundary layer triggers to increase when the fluid enters the tube in the walls of the walls having a temperature distinct from that of the fluid. The developed thermal condition is achieved after the flow passes a certain position.

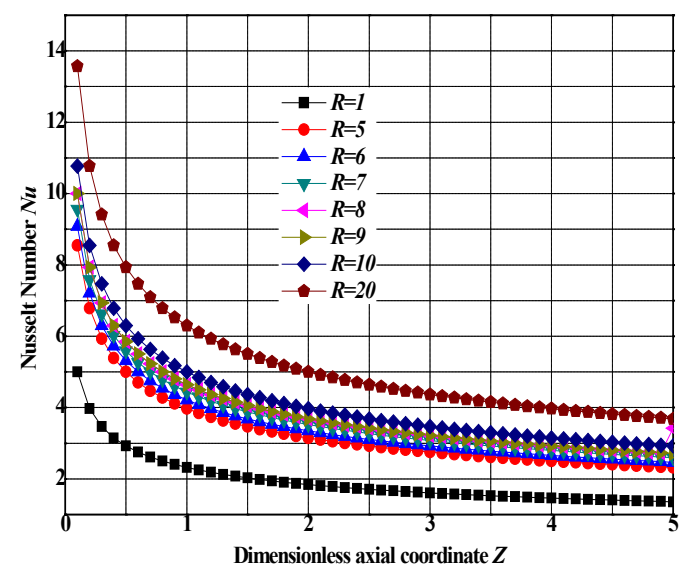

Figure 7. Nusselt number as a function of axial position for different tube radius

Fig. 8 shows the Nusselt number as a function of the longitudinal coordinates for different values of the Peclet number. It is observed that the increase in the number of Peclet leads to an increase in the number of Nusselt. As can be seen, the Péclet number has a much more pronounced effect on the Nusselt values for positions near the tube entrance. However the curve exhibits the same overall behavior - larger $\mathrm{Nu}$ at small $\mathrm{Z}$ and more or less constant value of large $Z$. In the tube entry region, where the boundary layer has expanded, we can see the reduction of the Nusselt number where it stabilizes in the fully developed thermal zone to a constant value does not depend on the Reynolds number and the heat flux. Hence, the thermal coefficient (h) appeared unlimited at the birth of the thermal boundary layer, and then gradually decreases to a stable value when the flow is fully developed at the origin. The numerical results clearly illustrate that the value of the Nusselt number increases and then decreases sharply over the entire longitudinal position of the tube.

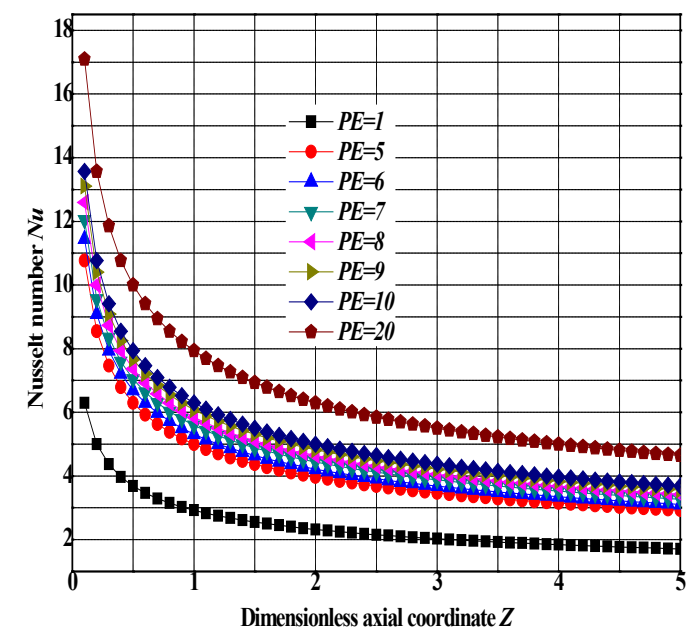

Figure 8. Nusselt number as a function of axial position with various Peclet numbers

\subsection{Comparative results with earlier results}

We found deeply in the specialized literature, the work of Shah and London [16] where they presented the numerical solutions of the well-known Nusselt-Graetz problem of heat transfer to an incompressible fluid with constant properties flowing through a circular duct having a uniform wall temperature and a fully developed laminar velocity profile. The asymptotes for the local Nusselt number correlation in the thermal entrance region of a circular tube.

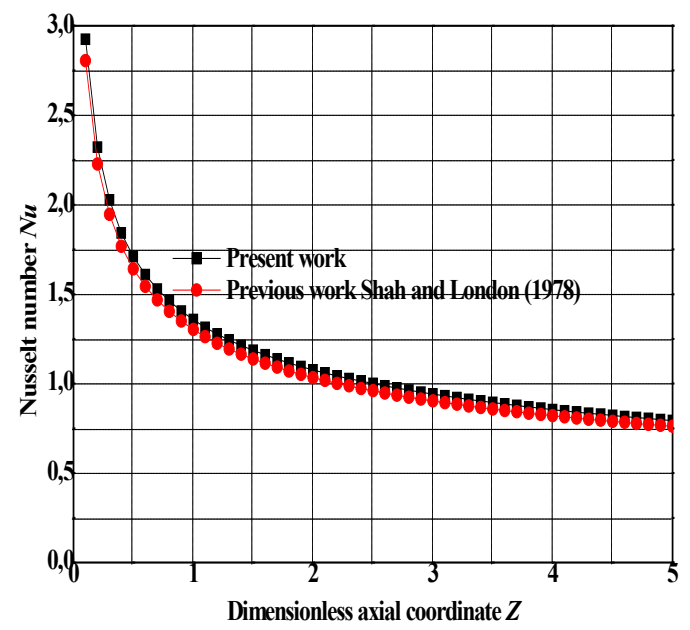

Figure 9. Validation of the present analytical model against the data literature value obtained by Shah and London [16] 
Fig. 9 plots the present analytical results for Nusselt number versus $Z$ at the thermal entrance region of circular conduit and comparison to existing literature correlations established by Shah and London [16]. These results are presented in this figure, where the Nusselt number is plotted against the axial coordinate $Z$. Examination of this one reveals the expected trend whereby higher Nusselt numbers correspond to very small values of the axial position $\mathrm{Z}$ at the entrance of the tube. It has been found that for the unblocked tube, the local Nusselt number decreases gradually as $\mathrm{Z}$ increases. From the figure 9, it can be seen that the results from both the analytical and the previous work are very similar which implies that the present work with the analytical method gives us a good correlation with the investigations found in the literature. Given the sources of variability of the Nusselt number calculated in the input thermal region, this result is considered satisfactory.

\section{CONCLUSION}

This paper presented an analytical and numerical solution to the Levèque approximation problem in order to predict the evolution of the thickness of the boundary layer as well as the temperature of the fluid at thermal entrance fully developed region through a circular tube with boundary condition at the axial coordinate origin. The exact solution methodology was based on the similarity variable and the generalized integral transform technique while the numerical approach is based on the integration technique of two differential equations with the Runge Kutta method of order 4 (RK4) programmed in Visual FORTRAN v5.0. The solution method was verified to lead to converging values which are in accordance with physically expected results. After demonstrating the convergence of the solution, the Nusselt number distribution of different Péclet values was analyzed, and the results are also in accordance with expected literature values. As a conclusion, we can say that both the simulated and data results that were found in the literature on the correlation of the Nusselt number are quite in good agreement. As final comments one should mention that the same solution procedure can be used for any dynamically developed velocity profile, as it occurs in many other occasions. Also, the methodology can be easily extended to other configurations such as another channel geometries, different wall heating conditions, and vicious and other flow heating effects.

\section{REFERENCES}

[1] Manoj KT, Rajsekhar P. (2017). Numerical analysis of natural convection in a triangular cavity with different configurations of hot wall. International Journal of Heat $\begin{array}{lll}\text { and } & \text { Technology } & 35(1) \text { : }\end{array}$ https://doi.org/10.18280/ijht.350102

[2] Muhammad A, Aftab H, Syed HA, Ali MA, Muizz PM. (2017). Simulation of corrugated plate heat exchanger for heat and flow analysis. International Journal of Heat $\begin{array}{lll}\text { and } & \text { Technology } & \text { 205-210. }\end{array}$ https://doi.org/10.18280/ijht.350127

[3] Hamad MAA, Ferdows M. (2012). Similarity solutions to viscous flow and heat transfer of nanofluid over nonlinearly stretching sheet. Applied Mathematics and
Mechanics (English Edition) 33(7): 923-930.

[4] Gulotta TM, Guarino F, Cellura M, Lorenzini G. (2017). Constructal law optimization of a boiler. International Journal of Heat and Technology 35(2): 297-305. https://doi.org/10.18280/ijht.350210

[5] Boukhalkhal AL, Lasbet Y, Makhlouf M, Loubar K. (2017). Numerical study of the chaotic flow in threedimensional open geometry and its effect on the both fluid mixing and heat performances. International Journal of Heat and Technology 35(1): 1-10. https://doi.org/10.18280/ijht.350101

[6] Ambethkar V, Kumar M. (2017). Numerical solutions of 2-D unsteady incompressible flow with heat transfer in a driven square cavity using stream function-vorticity formulation. International Journal of Heat and Technology 35(3): 459-473. https://doi.org/10.18280/ijht.350303

[7] Wei DM, Al-ashhab S. (2014). Similarity solutions for non-Newtonian power-law fluid flow. Applied Mathematics and Mechanics (English Edition) 35(9): $1155-1166$

[8] Trîmbijas R, Grosan T, Pop I. (2015). Mixed convection boundary layer flow past vertical flat plate in nanofluid: Case of prescribed wall heat flux. Applied Mathematics and Mechanics (English Edition) 36(8): 1091-1104.

[9] Ahmed SE. (2017). Modeling natural convection boundary layer flow of micropolar nanofluid over vertical permeable cone with variable wall temperature. Applied Mathematics and Mechanics (English Edition) 38(8): 1171-1180.

[10] Shen L, Lu C. (2017). Mechanism of three-dimensional boundary-layer receptivity. Applied Mathematics and Mechanics (English Edition) 38(9): 1213-1224.

[11] Mahanthesh B, Gireesha BJ, Shehzad SA, Abbasi FM, Gorla RSR. (2017). Nonlinear three-dimensional stretched flow of an Oldroyd-B fluid with convective condition, thermal radiation, and mixed convection. Applied Mathematics and Mechanics (English Edition) 38(7): 969-980.

[12] Eldesoky IM, Abdelsalam SI, Abumandour RM, Kamel MH, Vafai K. (2017). Interaction between compressibility and particulate suspension on peristaltically driven flow in planar channel. Applied Mathematics and Mechanics (English Edition) 38(1): 137-154.

[13] Baehr H, Stephan K. (1998). Heat Transfer, SpringerVerlag.

[14] Stephan K. (1959). Warmeubergang und druckabfall bei nicht ausgebildeter laminar stromung in rohren und in ebenen spalten. Chemie-Ingenieur-Technik 31(12): 773778.

[15] Asako Y, Nakamura H, Faghri M. (1988). Developing laminar flow and heat transfer in the entrance region of regular polygonal ducts. International Journal of Heat Mass Transfer 31(12): 2590-2593.

[16] Shah RK, London AL. (1978). Laminar flow forced convection in ducts. Academic Press, New York, NY.

[17] Kakac S, Shah RK, Aung W. (1987). Handbook of Single Phase Convective Heat Transfer, Wiley, New York.

[18] Ebadian MA. Dong ZF. (1998). Forced convection internal flows in ducts. In: Handbook of heat transfer, 3rd Edition, McGraw-Hill, New York.

[19] Kakac S, Yener Y. (1983). Laminar forced convection 
in the combined entrance region of ducts. Low Reynolds Number Heat Exchangers,S.Kakac, R. K. Shah and A. E. Bergles, eds, Hemisphere Publishing, Washington, 165-204.

[20] Hausen H. (1943). Darstellung des Wärmeübergangs in Rohren durch verallgemeinerte Potenzbezie-hungen. VDI-Zeitung, Suppl. Verfahrenstechnik 4: 91-98.

[21] Churchill SW, Ozoe H. (1973). Correlations for laminar forced con-vection with uniform heating in flow over a plate and in developing and fully developed flow in a tube. ASME J. Heat Transfer 95(1): 78-84.

[22] Churchill SW, Ozoe H. (1973). Correlations for laminar forced con-vection in flow over an isothermal flat plate and in developing and fully developed flow in an isothermal tube. ASME J. Heat Transfer 95(3): 416-419.

[23] Yilmaz T, Cihan E. (1993). General equation for heat transfer for laminar flow in ducts of arbitrary crosssections. International Journal of Heat and Mass Transfer 36(13): 3265-3270.

[24] Yilmaz T, Cihan E. (1995). An equation for laminar flow heat transfer for constant heat flux boundary condition in ducts of arbitrary cross-sectional area. J. Heat Transfer 117(3): 765-766.

[25] Belhocine A, Wan Omar WZ. (2016). Numerical study of heat convective mass transfer in a fully developed laminar flow with constant wall temperature. Case Studies in Thermal Engineering 6: 116-127.

[26] Belhocine A. (2016). Numerical study of heat transfer in fully developed laminar flow inside a circular tube. International Journal of Advanced Manufacturing Technology Int J AdvManuf Tech 85(9): 2681-2692.

[27] Belhocine A, Wan Zaidi WO. (2017). Exact Graetz problem solution by using hypergeometric function. International Journal of Heat and Technology 35(2): 347-353. https://doi.org/10.18280/ijht.350216

[28] Rajput GR, Krishnaprasad JSVR, Timol MG. (2016). Group theoretic technique for mhd forced convection laminar boundary layer flow of nanofluid over a moving surface. International Journal of Heat and Technology 34(1): 1-6. https://doi.org/10.18280/ijht.340101

[29] Bird RB, Stewart WE, Lightfoot EN. (1960). Transport Phenomena. John Wiley and Sons, New York.

[30] Subramanian SR. (2012). The Graetz Problem. Web. 12 Apr 1-13.

[31] Newman J. (1969). Extension of the Lévêque solution, Trans. ASME J. Heat Transfer 91(1): 177-1978.

[32] Abramowitz M. Stegun I. (1965). Handbook of mathematical functions. Dover, New York.

\section{NOMENCLATURE}

$\begin{array}{ll}{[a, b]} & \text { Internal of integration of PDE's } \\ C_{p} & \text { Heat capacity, } \mathrm{Jkg}^{-1} \mathrm{~K}^{-1} \\ C_{n} & \text { Coefficient of solution defined in Eq.(8) } \\ F & \text { Temperature field function which is the } \\ & \text { solution of Eq.(18) } \\ f(x, y, z) & \text { Function defined in two general 1st order } \\ & \text { ODE's } \\ G_{n} & \text { Eigenfunctions of a proper Sturm-Liouville } \\ & \text { system } \\ g(x, y, z) & \text { Function defined in two general 1st order } \\ & \text { ODE's }\end{array}$

$k$

$k_{0}$

$k_{1}$

$k_{2}$

$k_{3}$

$l_{0}$

$l_{1}$

$l_{2}$

$l_{3}$

$h$

$h$

$N$

$\mathrm{Nu}$

$\mathrm{Pe}$

$q_{\omega}$

$R$

$\operatorname{Re}$

$r, \theta, z$

$T$

$T_{b}$

$T_{0}$

$T_{\omega}$

$t$

$x$

$X$

$Y$

$y_{i}, y_{i+1}$

$u_{r}, u_{\theta}, u_{z}$

V

$v_{0}$

Z

$z$

$z_{i}, z_{i+1}$

\section{Greek symbols}

$\alpha$
$\beta_{n}$
$\Gamma$
$\delta$
$\theta$
$\rho$
$\mu$
$\eta$

Subscripts

RK04
Thermal conductivity, $\mathrm{Wm}^{-1} \mathrm{~K}^{-1}$

Increment based on the slope at the beginning of the interval

Increment based on the slope at the midpoint of the interval

Increment based on the slope at the midpoint of the interval

Increment based on the slope at the end of the interval

Increment based on the slope at the beginning of the interval

Increment based on the slope at the midpoint of the interval

Increment based on the slope at the midpoint of the interval

Increment based on the slope at the end of the interval

Heat transfer coefficient, $\mathrm{Wm}^{-2} \mathrm{~K}^{-1}$

Step size

Number of steps

Nusselt number

Peclet number

Heat flux from fluid wall, $\mathrm{Wm}^{-2}$

Tube radius, $\mathrm{m}$

Reynolds number

Cylindrical coordinates, $\mathrm{m}$

Temperature of the fluid inside a circular tube, $\mathrm{K}$

Bulk temperature, $\mathrm{K}$

Temperature of the fluid entering the tube, $\mathrm{K}$

Temperature of the fluid on the wall of the tube, $\mathrm{K}$

Time, $\mathrm{s}$

Transversal coordinate, $\mathrm{m}$

Dimensionless radial direction, $\mathrm{m}$

Dimensionless radial direction, $\mathrm{m}$

Values of $\mathrm{y}$ at $x_{i}$ and $x_{i+1}$ respectively

Cartesian velocity components, $\mathrm{ms}^{-1}$

Fluid longitudinal velocity, $\mathrm{ms}^{-1}$

Maximum axial velocity of the fluid, $\mathrm{ms}^{-1}$

Dimensionless axial direction

Longitudinal coordinate (m)

Values of $z$ at $x_{i}$ and $x_{i+1}$ respectively

Thermal diffusivity, $\mathrm{m}^{2} \mathrm{~s}^{-1}$

Eigenvalues

Gamma function

Thickness of the thermal boundary layer, $\mathrm{m}$

Dimensionless bulk average temperature

Density of the fluid, $\mathrm{kgm}^{-3}$

Dynamic viscosity of the fluid, $\mathrm{kgm}^{-1} \mathrm{~s}^{-1}$

Similarity variable

4th order Runge-Kutta method 